\title{
Explorations of Post Constrained Recovery Residual Stress of Shape Memory Alloys in Self-healing Applications
}

\author{
Muhammad Istiaque Haider, Maysam Rezaee, Nathan Salowitz \\ Advanced Structures Laboratory, Mechanical Engineering Department, \\ University of Wisconsin Milwaukee, Milwaukee, Wisconsin, United States
}

\begin{abstract}
Self-healing materials with intrinsic capabilities of geometric restoration and damage recovery have a tremendous potential to improve product safety and reliability, especially in space applications where recovery or manual performance of repairs may be prohibitive, dangerous, or impossible. Selfhealing materials typically incorporate a complex internal structure containing constituent materials of different functionality and one of the primary methods is to reinforce self-healing materials with shape memory alloys that can be activated to restore geometry and close a fracture. Recent experimental investigations revealed that Nickel Titanium (NiTi) shape memory alloys (SMAs) could repeatedly produce stable residual stresses following constrained recovery when held in a constrained condition during temperature change through the forward and reverse transformations. The ability to produce this post constrained recovery residual stress (PCRRS) in a low temperature state, without continuous actuation, and to regenerate it repeatedly have the potential to advance selfhealing capabilities and even damage prevention.
\end{abstract}

\section{Introduction}

Functionality, durability, and longevity of mechanical structures are consistent considerations in design. In standard engineering practice, structures are designed with material selection to prevent the occurrence of critical damage within a service lifetime. Recently, there has been a huge interest in materials that can self-heal, as this property can potentially increase materials lifetime, reduce replacement costs, and improve product safety and reliability (Aïssa, Therriault, Haddad, \& Jamroz, 2012). The design philosophy is based on a material not just tolerating damage but having an innate ability to rectify (heal) the damage and restore product function to acceptable levels (higher than 70\% initial functionality level) (Alaneme \& Omosule, 2015; Hayes, Jones, Marshiya, $\&$ Zhang, 2007). Self-healing materials have the potential to supports long duration space flights where manual repair is dangerous or not possible. This study introduces a new technique for designing and developing self-healing materials utilizing post constrained recovery residual stresses (PCRRS) observed in nickel titanium (NiTi) shape memory alloys (SMAs) while undergoing constrained recovery. Based on the recent experimental findings, NiTi SMAs showed the ability to generate residual stresses after undergoing constrained recovery and returning to a low temperature, martensitic state while being constrained. This has the potential to be useful in applications like self-healing materials where loads are desirable without the continuous application of heat or energy. This paper represents phenomenological exploration of experiments that were conducted to understand mechanisms of PCRRS and discuss its potential application scope and benefits in a self-healing material design regime, especially as applicable to space applications.

1.1 Motivation Self-healing materials, inspired by biological materials, have the ability to repair themselves and to recover functionality using the resources inherently available to them (White, et al., 2001), which could lessen the burden of periodic inspection in damage monitoring, reduce cost of product maintenance and replacement, and frequency of shutdown due to product 
failure in service (Trask, Williams, \& Bond, 2007). Mimicking of the biological process of healing in inorganic systems can be considered a herculean task; however, most of the research efforts were based on non-metallic materials for easy implementation.

Major applications of self-healing materials include spacecraft and aerospace structures, automotive structures and defense industries (Das, Melchior, \& Karumbaiah, 2016), hence, development of these self-healing materials gained significant attention and researchers have been working on this topic for several decades.

Metallic self-healing composites are of great practical interest because their structural properties can be superior to polymer and ceramic based self-healing materials (Ferguson, Schultz, \& Rohatgi, 2014). This is primarily due to the common approach of encapsulating liquid resin or adhesives in polymer and ceramic self-healing materials. The concept is that damage would release the liquid that would flow into the damage and adhere the structure back together. Challenges with this approach are that the encapsulated liquid adds weight to the structure while not providing any strength until released and after release, voids are left behind. Further, polymers and liquid adhesives generally are not well suited to the range of extreme temperatures that can be experienced in space flight. Metal matrix self-healing composites are more temperature tolerant and have the advantage of being entirely solid and therefore load bearing.

Two common healing modes of self-healing mechanisms are bulk shape restoration, and smallscale crack filling and bonding. Bulk shape restoration is typically achieved by incorporating shape memory fiber reinforcements into structures, forming a sparse fiber composite (Salowitz, et al., 2018). NiTi can be implemented as an actuator utilizing its distinctive shape memory effect and superelastic properties where it is deformed in its low temperature, martensitic state and then heated through a reversible austenite phase transformation. This property is utilized comprehensively in self-healing materials to close small cracks and large fractures. NiTi wires embedded into the matrix experience deformation when the matrix fractures. When the structure is heated the NiTi fibers are actuated and restore the bulk geometry, closing cracks. Prior to the work of this lab, the NiTi was embedded in its parent, undeformed geometry. As bulk restoration has been limited to moving the crack faces to adjacent position, no compressive loads were developed across the crack face during recovery.

With the ability to withstand over 5\% strain from its parent geometry in its low temperature martensitic state, if NiTi is inhibited from returning to its preferred parent geometry while in a high temperature, austenitic state, it can produce large stresses. This is known as constrained recovery. These large stresses are only produced while the NiTi was kept in its high temperature austenite state requiting continuous thermal actuation. Recent experimental investigation has revealed a phenomenon wherein the material can be actuated to a high temperature state, constrained from returning to its original geometry, and upon ceasing actuation and returning to a low temperature, the material will continue to exert a force on the constraint. This property has been named post constrained recovery residual stresses (PCRRS) (Haider, Rezaee, Yazdi, \& Salowitz, 2019; Haider, Yazdi, Rezaee, Tsai, \& Salowitz, 2019; Haider, Correa, Moghadam, Yan, $\&$ Salowitz, 2018). This capability provides a new way for the material to be used as an actuator that may be beneficial to previous applications like self-healing material and could support new applications like using a dispersion of NiTi particles to inhibit fatigue cracking in material structures. This property will allow for predictive design of self-healing materials, which produce residual crack closing loads that can resist external loads. Extended experiments confirmed the 
ability to produce PCRRS without continuous delivery of energy, which could direct towards new competency in smart material and structure design. This research aimed to represent the concept of using PCRRS in developing self-healing metallic materials specially for creating constant crack closing load without continuous application of energy.

1.2 Background. Self-healing materials are typically categorized in two ways: healing mechanism and autonomy (Blaiszik, et al., 2010). Considering the properties of their healing mechanism, self-healing materials are categorized (Blaiszik, et al., 2010; Kilicli, Yan, Salowitz, \& Rohatgi, 2018) into autonomic self-healing materials, which are self-actuating and do not require external stimuli, and non-autonomic self-healing materials require external stimulus, such as application of heat, light, or a catalyst, in order to heal (Burton, Gao, \& Brinson, 2006; Chen, Hehr, Dapino, \& Anderson, 2015; Misra, 2013). Small-scale crack filling and bonding is most often autonomic and accomplished with encapsulated liquid bonding agents that are released upon damage, flow into cracks, and then solidify (Misra, 2013). Bulk shape restoration is generally non-autonomic and is typically accomplished using shape memory reinforcements embedded within the material that require heating to actuate healing. Bulk shape restoration is also capable of recovering from large fractures where the matrix has been completely separated. Bulk shape restoration and immobilization of the pieces relative to each other must happen before bonding for proper healing to occur. Non-autonomic self-healing mechanisms are beneficial in this regard as the order of actuation can be controlled.

The functional properties of SMA such as SME and pseudoelasticity originate from thermoelastic martensitic phase transformations between austenite and martensite phases (Lagoudas, 2008). The SME refers to the ability of a SMA to undergo deformation at one temperature, and then recover its original, undeformed shape upon heating above its reverse transformation temperature. Recovery stress appears when this shape recovery is restrained during heating (Haider, Rezaee, Yazdi, \& Salowitz, 2019; Haider, Yazdi, Rezaee, Tsai, \& Salowitz, 2019; Haider, Correa, Moghadam, Yan, \& Salowitz, 2018). This recovery stress is used in many applications, such as actuators, SMA pipe couplings, robotic applications, and SMA composites (Kapgan \& Melton, 1990; Yamauchi, Ohkata, Tsuchiya, \& Miyazaki, 2011). The integration of SMAs into composite structures has resulted in many benefits, which include actuation (Song, Kelly, \& Agrawal, 2000), vibration control (Lau, 2002), damping (Li, Zhang, Xiong, \& Mai, 2010), sensing (Jani, Leary, Subic, \& Gibson, 2014), energy dissipation (Farahi, Esfahani, \& Sabzi, 2020) and self-healing (Hassan, Mehrpouya, Emamian, \& Sheikholeslam, 2013).

Very few relevant studies exist in the field of metallic self-healing studies incorporated with SMAs. Dutta, et al. (Dutta, et al., 2004; Wang, Dutta, \& Majumdar, 2006; Dutta, Pan, Ma, Majumdar, \& Harris, 2006) investigated the thermo-mechanical cycling response of Tin-SilverCopper $(\mathrm{SnAgCu}, \mathrm{Sn}-3.8 \% \mathrm{Ag}-0.7 \% \mathrm{Cu}$ ) solder reinforced with 11 vol. \% single NiTi fiber and 5 vol. \% NiTi particulates. These studies reduced inelastic strain range by $25 \%$; however, the healing process of cracks and the effect of a volume fraction of reinforcement on property variation were not studied. Manuel and Olson (Manuel \& Olson, 2007) fabricated a proof-of-concept composite (Sn-13at.\%Bi) with uniaxial oriented 1\% NiTi reinforcement resulted into recovery of $95 \%$ ultimate strength; however, this was offset by a reduction in uniform ductility from $6.4 \%$ to $2.2 \%$ attributed to defects that accumulated at the crack surface during healing. Expanded work of Manuel (Manuel M. , 2007) on healing in magnesium-based matrix (Mg5.7at.\%Zn2.7at.\%Al) with same volume fraction of NiTi as previous studies revealed that partial crack closure after healing; however, a $160 \%$ increase in uniform ductility resulted in composite toughening in the specimen. 
Furthermore, these studies did not delve into high volume fractions of NiTi reinforcement. Coughlin et al. (Coughlin J., Williams, Crawford, \& Chawla, 2009; Coughlin, Williams, \& Chawla, 2009) studied the strain recovery behavior of a Sn-3.5Ag alloy reinforced with 2-10\% continuous austenitic NiTi wire. Ruzek (Ruzek, 2009) found poor wettability between as-received and $\mathrm{Cu}$-coated NiTi SMA short fibers and the solder matrix. Misra's approach was to resolve these issues by forming off-eutectic alloys with higher percentages of NiTi volume aiming to recover high plastic strain/shape and healing of micro-cracks to develop high strength bonding. This study resulted in $92 \%$ of flexural strength recovery for Sn-20\% Bi /NiTi composite and $88 \%$ of the strength recovery for $\mathrm{Bi}-10 \% \mathrm{Sn} / \mathrm{NiTi}$ composite after healing.

Residual loads developed in constrained recovery process is exploited in different actuation applications, however, this actuation needs continuous application of energy. Actuation without continuous exertion of energy may be more efficient in many applications, especially in selfhealing materials or any pre-stressed structures. The residual load generated after undergoing constrained recovery and thermal actuation has the potential to be beneficial as this load can be repeatedly generated without continuous application of heat energy.

\section{Methods}

Initial sample preparations and experimental procedures to generate PCRRS via thermomechanical loading were reported by Salowitz et al (Salowitz, et al., 2018). Following exposure to PCRRS states, multiple extended thermomechanical experiments were executed to assess the behavior and to evaluate the consistency of the property over different loading paths. Preliminary experimentation initiated with the repeated application and withdrawal of the load identical to the maximum prestrain magnitudes following a thermal cycling to create and reproduce PCRRS. Afterward, the same experiments were reiterated with small strain of $0.5 \%$ loading without thermal cycling as PCRSS can be gained continually for every cycle of small strain application. These two experiments and subsequent loading procedures were presented thoroughly in Haider et al (Haider, Rezaee, Yazdi, \& Salowitz, 2019; Haider, Correa, Moghadam, Yan, \& Salowitz, 2018; Haider, Yazdi, Rezaee, Tsai, \& Salowitz, 2019). Furthermore, a circumstantial analysis was reported on magnitudes and trends of the recurring PCRRS due to both large and small strain cyclic applications. These studies were expanded by detailed analysis of the effect of training on the phenomena incorporating similar loading paths in trained samples. Additionally, a panoramic study of the process of repeated regeneration of PCRRS from decaying magnitudes over repeated small strain applications was rendered by Haider et al. (Haider, Rezaee, Yazdi, \& Salowitz, 2019).

This paper aims to review the experimental exploration that introduces and expands to understand mechanisms of post constrained recovery residual stress (PCRRS) and discuss its potential application scope and benefits in self-healing material development. Moreover, a new design is proposed to develop a self-healing metallic material incorporating PCRRS with efficient bulk restoration capabilities along with steady crack closing load without continuous application of energy.

All experiments with NiTi wires were implemented using TA instruments Q800 Dynamic Mechanical Analyzer (DMA) machine with integrated capacity of thermomechanical load application and recording outputs. Applied strain rates and heat application rates are kept consistent across the entire research paper. Samples were prepared by exposing to heating and 
subsequent cooling cycles in unconstrained conditions to attain the parent geometry in martensitic states.

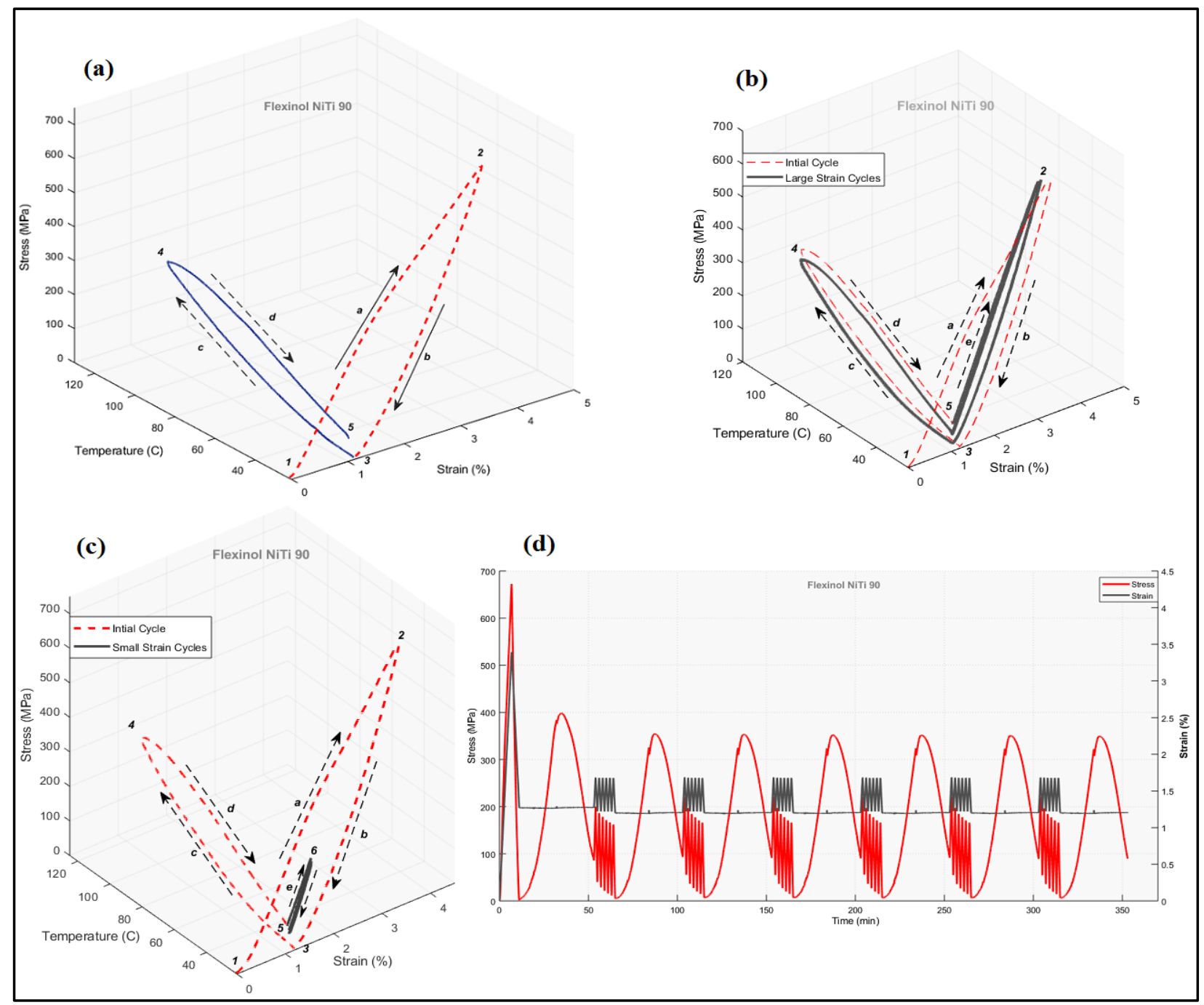

Fig. 1 Plot of NiTi wires exposed to different loading conditions. (a) Initial cycles to generate the PCRRS, (b) Wires exposed to the larger strain loading, (c) Wires exposed to the small strain loading, and (d) Sequential regeneration of PCRRS following thermal cycles after load application (Haider, Rezaee, Yazdi, \& Salowitz, 2019).

2.1 Initial cycle and experimentation with different loading. As shown in the figure 1(a), NiTi wire samples, from its self-accommodated twinned martensite states at point 1 , is loaded to the prestrain value of $3.4 \%$ at rate of $0.5 \%$ strain $\mathrm{mm}^{-1}$ following the path a to point 2 , then the wire is unloaded in the same rate as depicted by path $\mathrm{b}$ until it reached to the point 3 and later keeping constrained at point 3 , the sample was exposed to a thermal cycle at a rate of $5{ }^{\circ} \mathrm{C} \mathrm{min}-1$ along the path $\mathrm{c}$ and path $\mathrm{d}$ forcing the NiTi samples to a transition from martensite to austenite and then back to martensitic phase. At point 5, a residual load is observed in low temperature martensitic states.

From PCRRS state at point 5, the samples initially underwent two loading profiles. Firstly, it was exposed to large strain corresponding to maximum prestrain value of $3.4 \%$ mimicking the path $\mathrm{e}$ and removed the load following path $\mathrm{b}$ as shown figure 1(b). Afterwards, remaining in same 
displacement control, the temperature was increased to reach austenite and cooled to martensite resulted in consistent generation of PCRRS at point 5 for repeated cycles where a monotonic but decreasing trend in PCRRS value were observed over each cycle of large strain. Later, another set of experiments were done from point 5 with the repeated application and removal of small strain of $0.5 \%$ through path e as shown in the figure 1(c). It was observed that, these strains performed elastically and nearly linearly, validating that PCRRS would persists after exposure to small strain cycle, however, PCRRS magnitudes showed a monotonic decreasing trend again. At the initial stages of the experiments, there was an approximation that the monotonic reduction in PCRRS values upon loading may cause due to the lack of training of the materials. Training NiTi did not significantly vary the characteristics compared to the untrained NiTi.

2.2 Regeneration of PCRRS. Following the different loading paths and studies with trained materials, other experiments are performed to determine if the initial PCRRS could be restored after exposure to cyclic loading and the associated reduction in magnitude. In these experiments, following the thermomechanical load application of initial PCRRS generation, a sequence of multiple small strain was applied to the wires and exposure to the gradual reduction of PCRRS in every cycle and then a thermal load at a constrained state beyond Af and then returned to Mf, would recreate the PCRRS as identical to initial one. These sequences of small strain loading followed by thermal actuation were repeated multiple times as shown in figure 1 (d). Each sequence resulted in the regeneration of the PCRRS. Successive sequences of small strain applications confirm the regeneration of the PCRRS to its original value with small variation in magnitudes. First two sequences is seen to be in the highest variation in PCRRS which is less than $10 \%$ whereas a stabilized trend spotted in the remaining sequences.

2.3 Concept of Self-healing material with PCRRS. As discussed earlier, shape memory alloy (SMA) wires are reinforced inside the base alloy matrix to pull together crack surfaces and then bond the damaged surfaces together using the partial melting techniques. An off-eutectic alloy has been selected for fabricating the self-healing samples as these alloys have distinguished solidus and liquidus temperatures; and between these temperatures range, an off-eutectic alloy combination prevail as a semisolid paste of solid particles surrounded by molten liquid above a certain temperature. The amount of molten material can be controlled by heating to a specified temperature. Tin $(\mathrm{Sn})$ and Bismuth (Bi) form one such binary eutectic with a low melting point of $139{ }^{\circ} \mathrm{C}$ that has been investigated in SMA-based healing studies pioneered Manuel and Olsen (Manuel \& Olson, 2007) and further detailed studies were conduct by Misra (Misra, 2013) where a different combination of Sn-Bi alloys was fabricated to check the healing efficiency and bonding with NiTi wires and its effect on healing for different alloy composition of Tin and Bismuth. As this proposed self-healing material is still in conceptual phase, Sn-20\% Bi off-eutectic alloy of 165 ${ }^{\circ} \mathrm{C}$ healing temperature is selected as base matrix alloy to demonstrate the mechanism and to gain known crack bonding efficiency. Samples will be prepared by following the work done by Misra, where after making the alloy with known combinations, NiTi wires were etched and fluxed to remove $\mathrm{TiO}_{2}$ layer ensuring proper bonding with the base matrix. After preparing alloy and wires, a pressure infiltration technique adapted from Ruzek et al. (Ruzek, 2009) will be used to fabricate composites with a $20 \%$ volume fraction of prestrained reinforcement. As presented in the schematic shown in figure 2 , after exposing the matrix composite to a complete crack using the tensile tester, the specimens were removed from the tensile testing apparatus and heat-treated at $165^{\circ} \mathrm{C}$ for $24 \mathrm{~h}$ in vacuum to heal the crack. As in practical applications, healed specimen will be 
exposed to smaller load to simulate fatigue cracking in the specimen and as demonstrated before, due to PCRRS, material will be forced to obstruct the fatigue cracking load.
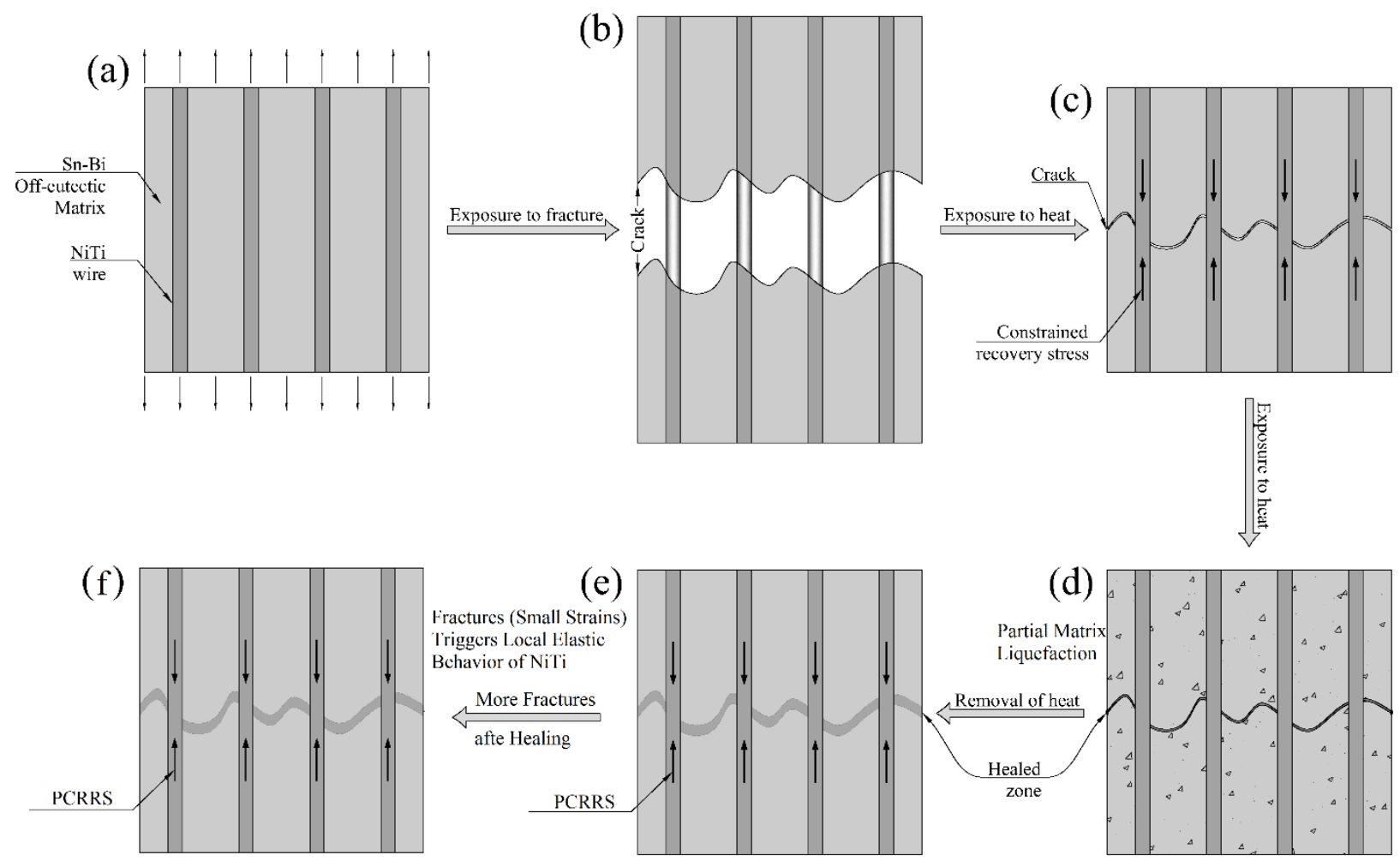

Fig. 2 Schematic overview of the self-healing process in metal/SMA composite with PCRRS. (a) Sn-Bi off-eutectic matrix reinforced with pre-strained NiTi wires exposed to tensile load, (b) Crack formation due to tensile load resulting in detwinning of the NiTi wires, (c) Compressive stress due constrained recovery after exposing to heat resulted into crack closure, (d) Adjacent crack face healing and bonding due partial melting, (e) Removal of heat resulted into PCRRS which creates residual load to inhibit cracks, and (f) Small strain application due to fatigue (or any service load) creates the blocking force due to PCRRS without heat application which obstruct further crack propagation of healed specimen.

\section{Discussion and Concluding Remarks}

Biomimetic self-healing material is a relatively new area in engineering research and application. The capacity of creating fracture closure load with bonding capabilities and to create residual load to inhibit further fracture generating load from fatigue or any other form of stress would be a large step toward the greater goals of preventing catastrophic structural failures and prolonging the life of structures. The approaches presented in this paper are still at conceptual and elementary levels. As self-healing materials can be potentially extremely beneficial for a wide range of aerospace and aviation applications, presently research in this area is extremely appealing. This has been evidently shown that NiTi wires have the ability to generate PCRRS and this ability to actuate without the application of energy will lead to a paradigm shift in intelligent material design. Another application of PCRRS that might create colossal effect in research would be to use this as a dispersion in structural materials to generate residual compressive stresses in the host material, intended to inhibit fatigue crack formation or growth that occurs with cyclic loading. Research is 
ongoing to characterize and to develop the theoretical modeling of PCRRS to predict the behavior in order to implement it in engineering applications.

\section{Acknowledgements}

Support for this research was provided by:

- The Support for Undergraduate Research Fellows (SURF) program at the University of Wisconsin-Milwaukee

- Funds from University of Wisconsin - Milwaukee Department of Mechanical Engineering

- Dynalloy through their SMArt program

- SAES Getters S.p.A.- SAES® Smart Materials (SSM)

\section{References}

Aïssa, B., Therriault, D., Haddad, E., \& Jamroz, W. (2012). Self-Healing Materials Systems: Overview of Major Approaches and Recent Developed Technologies. Advances in Materials Science and Engineering, 2012. doi:https://doi.org/10.1155/2012/854203

Alaneme, K., \& Omosule, O. (2015). Experimental Studies of Self Healing Behaviour of UnderAged Al-Mg-Si Alloys and 60Sn-40Pb Alloy Reinforced Aluminium Metal-Metal Composites. Journal of Minerals and Materials Characterization and Engineering, 3(1), 1-8. doi:10.4236/jmmce.2015.31001

Blaiszik, B., Kramer, S., Olugebefola, S., Moore, J., Sottos, N., \& White, S. (2010). Self-Healing Polymers and Composites. Annual Review of Materials Research, 40(1), 179-211. doi:10.1146/annurev-matsci-070909-104532

Burton, D., Gao, X., \& Brinson, L. (2006). Finite element simulation of a self-healing shape memory alloy composite. Mechanics of Materials, 38(5-6), 525-537. doi:https://doi.org/10.1016/j.mechmat.2005.05.021

Chen, X., Hehr, A., Dapino, M., \& Anderson, P. (2015). Deformation Mechanisms in NiTi-Al Composites Fabricated by Ultrasonic Additive Manufacturing. Shape Memory and Superelasticity, 1, 294-309. doi:https://doi.org/10.1007/s40830-015-0032-1

Coughlin, J., Williams, J., \& Chawla, N. (2009). Mechanical behavior of NiTi shape memory alloy fiber reinforced Sn matrix "smart" composites. Journal of Materials Science, 44(3), 700707. doi:https://doi.org/10.1007/s10853-008-3188-7

Coughlin, J., Williams, J., Crawford, G., \& Chawla, N. (2009). Interfacial Reactions in Model NiTi Shape Memory Alloy Fiber-Reinforced Sn Matrix "Smart" Composites. Metallurgical and Materials Transactions A, 40, 176-184. doi:https://doi.org/10.1007/s11661-008-9676-1

Das, R., Melchior, C., \& Karumbaiah, K. (2016). Self-healing composites for aerospace applications. In S. Rana, \& R. Fangueiro, Advanced Composite Materials for Aerospace Engineering: Processing, Properties and Applications (pp. 333-364). Woodhead Publishing. doi:https://doi.org/10.1016/B978-0-08-100037-3.00011-0

Dutta, I., Majumdar, B., Pan, D., Horton, W., Wright, W., \& Wang, Z. (2004). Development of a novel adaptive lead-free solder containing reinforcements displaying the shape-memory 
effect. Journal of Electronic Materials, 33, 258-270. doi:https://doi.org/10.1007/s11664004-0131-9

Dutta, I., Pan, D., Ma, S., Majumdar, B., \& Harris, S. (2006). Role of shape-memory alloy reinforcements on strain evolution in lead-free solder joints. Journal of Electronic Materials, 35, 1902-1913. doi:https://doi.org/10.1007/s11664-006-0174-1

Farahi, B., Esfahani, M., \& Sabzi, J. (2020). Experimental Investigation on the Behavior of Reinforced Concrete Beams Retrofitted with NSM-SMA/FRP. Amirkabir Journal of Civil Engineering, 51(4), 133-136. doi:10.22060/ceej.2016.689

Ferguson, J., Schultz, B., \& Rohatgi, P. (2014). Self-Healing Metals and Metal Matrix Composites. The Journal of The Minerals, Metals \& Materials Society, 66, pages866-871. doi:https://doi.org/10.1007/s11837-014-0912-4

Haider, M., Correa, A., Moghadam, A., Yan, X., \& Salowitz, N. (2018). Experimental Exploration Of Post Constrained Recovery Mechanics Of NiTi. Proceedings of the ASME 2018 Conference on Smart Materials, Adaptive Structures and Intelligent Systems, (p. V002T02A012). San Antonio, Texas. doi:https://doi.org/10.1115/SMASIS2018-8168

Haider, M., Rezaee, M., Yazdi, A., \& Salowitz, N. (2019). Investigation into post constrained recovery properties of nickel titanium shape memory alloys. Smart Materials and Structures, 28(10), 105044. doi:https://doi.org/10.1088/1361-665X/ab3ad4

Haider, M., Yazdi, A., Rezaee, M., Tsai, L., \& Salowitz, N. (2019). Mechanics of Post Constrained Recovery Residual Stress Produced by NiTi. Proceedings of the ASME 2019 Conference on Smart Materials, Adaptive Structures and Intelligent Systems, (p. V001T02A007). Louisville, Kentucky. doi:https://doi.org/10.1115/SMASIS2019-5619

Hassan, M., Mehrpouya, M., Emamian, S., \& Sheikholeslam, M. (2013). Review of Self-Healing Effect on Shape Memory Alloy (SMA) Structures. Advanced Materials Research, 701, 8792. doi:https://doi.org/10.4028/www.scientific.net/AMR.701.87

Hayes, S., Jones, F., Marshiya, K., \& Zhang, W. (2007). A self-healing thermosetting composite material. Composites Part A: Applied Science and Manufacturing, 38(4), 1116-1120. doi:10.1016/j.compositesa.2006.06.008

Jani, J., Leary, M., Subic, A., \& Gibson, M. (2014). A review of shape memory alloy research, applications and opportunities. Materials \& Design, 56, 1078-1113. doi:https://doi.org/10.1016/j.matdes.2013.11.084

Kapgan, M., \& Melton, K. (1990). Shape memory alloy tube and pipe couplings. In T. Duerig, K. Melton, D. Stockel, \& C. Wayman, Engineering Aspects of Shape Memory Alloys (pp. 137148). London: Butterworth-Heinemann.

Kilicli, V., Yan, X., Salowitz, N., \& Rohatgi, P. (2018). Recent Advancements in Self-Healing Metallic Materials and Self-Healing Metal Matrix Composites. The Journal of The Minerals, Metals \& Materials Society, 70, 846-854. doi:https://doi.org/10.1007/s11837018-2835-y

Lagoudas, D. (2008). Shape Memory Alloys: Modeling and Engineering Applications. Springer US. 
Lau, K.-t. (2002). Vibration characteristics of SMA composite beams with different boundary conditions. Materials and Design, 23(8), 741-749. doi:https://doi.org/10.1016/S02613069(02)00069-9

Li, D., Zhang, X., Xiong, Z., \& Mai, Y.-W. (2010). Lightweight NiTi shape memory alloy based composites with high damping capacity and high strength. Journal of Alloys and Compounds, 190(1-2), L15-L19. doi:https://doi.org/10.1016/j.jallcom.2009.10.025

Manuel, M. (2007). Design of a Biomimetic Self-Healing Alloy Composite. Evanston, Illinois: PhD Thesis - Northwestern University.

Manuel, M. V., \& Olson, G. B. (2007). Biomimetic Self-Healing Metals. Proceedings of the 1st Intl. Conference on Self-Healing Materials. Noordwijk aan Zee.

Misra, S. (2013). Shape Memory Alloy Reinforced Self-healing Metal Matrix Composites. Milwaukee, Wisconsin: Master's Thesis-University of Wisconsin-Milwaukee. Retrieved from https://dc.uwm.edu/etd/731/

Ruzek, A. (2009). Synthesis and characterization of metallic systems with potential for selfhealing. Milwaukee, Wisconsin: Master's Thesis- University of Wisconsin-Milwaukee.

Salowitz, N., Correa, A., Santebennur, T., Moghadam, A., Yan, X., \& Rohatgi, P. (2018). Mechanics of nickel-titanium shape memory alloys undergoing partially constrained recovery for self-healing materials. Journal of Intelligent Material Systems and Structures, 29(15), 3025-3036. doi:https://doi.org/10.1177/1045389X18781260

Song, G., Kelly, B., \& Agrawal, B. (2000). Active position control of a shape memory alloy wire actuated composite beam. Smart Materials and Structures, 9(5), 711. doi:https://doi.org/10.1088/0964-1726/9/5/316

Trask, R., Williams, H., \& Bond, I. (2007). Self-healing polymer composites: mimicking nature to enhance performance. Bioinspiration \& Biomimetics, 2(1). doi:https://doi.org/10.1088/1748-3182/2/1/P01

Wang, Z., Dutta, I., \& Majumdar, B. (2006). Thermomechanical response of a lead-free solder reinforced with a shape memory alloy. Scripta Materialia, 54(4), 627-632. doi:https://doi.org/10.1016/j.scriptamat.2005.10.037

White, S., Sottos, N., Geubelle, P., Moore, J., Kessler, M., Sriram, S., . . Viswanathan, S. (2001). Autonomic healing of polymer composites. Nature, 409, 794-797. doi:https://doi.org/10.1038/35057232

Yamauchi, K., Ohkata, I., Tsuchiya, K., \& Miyazaki, S. (2011). Shape Memory and Superelastic Alloys: Applications and Technologies. Woodhead Publishing. 